\title{
Radiating stars with composite matter distributions
}

\author{
Sunil D. Maharaj ${ }^{\mathrm{a}}$ i, Byron P. Brassel $^{\mathrm{b}}$ \\ Astrophysics Research Centre, School of Mathematics, Statistics and Computer Science, University of KwaZulu-Natal, Private Bag 54001, \\ Durban 4000, South Africa
}

Received: 13 February 2021 / Accepted: 18 April 2021 / Published online: 27 April 2021

(C) The Author(s) 2021

\begin{abstract}
In this paper we study the junction conditions for a generalised matter distribution in a radiating star. The internal matter distribution is a composite distribution consisting of barotropic matter, null dust and a null string fluid in a shear-free spherical spacetime. The external matter distribution is a combination of a radiation field and a null string fluid. We find the boundary condition for the composite matter distribution at the stellar surface which reduces to the familiar Santos result with barotropic matter. Our result is extended to higher dimensions. We also find the boundary condition for the general spherical geometry in the presence of shear and anisotropy for a generalised matter distribution.
\end{abstract}

\section{Introduction}

A substantial amount of work on radiating stars in relativistic regimes has been undertaken in the standard Santos [1] framework. This construction addresses the question of matching a spherically symmetric interior matter distribution to the exterior radiating Vaidya spacetime across a comoving surface. This approach has been extended to include the electromagnetic field [2-5], the cosmological constant [6-9], the presence of nonzero shear [10-12] and dissipative effects [13-16]. Matching across a comoving surface is important in modeling a relativistic star in general relativity and in discussing the evolution of the system. A variety of exact solutions to the Einstein field equations, with a matter distribution that has to be necessarily heat conducting, and the nonlinear boundary condition have been found. Some of the resulting astrophysical models are given in the recent treatments [17-24]. The problem of matching general hypersurfaces and junction conditions, containing timelike, spacelike or null surfaces, was analysed by Mars and Senovilla [25]. The general matching of two spherically symmetric regions

\footnotetext{
a e-mail: maharaj@ukzn.ac.za (corresponding author)

b e-mail: drbrasselint@gmail.com
}

across a timelike hypersurface was completed by Fayos et al. [26]. Here we are interested in conditions arising in matching across a comoving surface. Also note that the matching conditions have been studied in particular modified theories, for example Olmo and Rubiera-Garcia [27] and Yousaf $[28,29]$ have considered matching and physical applications in $f(R)$ gravity theories.

We believe that the resulting boundary conditions, for our generalised matter distributions, will assist in analysing the radiative collapse dynamics of spacetimes with spherical topologies. A recent example with spherical, toroidal and higher genus topologies in gravitational collapse including the formation of trapped surfaces was completed by Mena and Oliveira [30]. Another interesting result is that of Charan et al. [31] who described charged anisotropic spherical collapse in the presence of heat flow where the dynamics are reduced to an ordinary differential equation. In this model the presence of charge delays the collapse of the star and the energy conditions are satisfied. The generalised matter distribution in our work should have interesting consequences on the physical features of the radiating stellar model.

The matter distribution in the treatment of Santos [1] was shear-free. Most studies take the interior matter distribution to be a barotropic fluid. Anisotropic stresses, viscosity and electromagnetic effects can also be part of the interior energy momentum tensor. Di Prisco et al. [15] showed that it is possible to also include null dust in the interior matter distribution of the star. The exterior of the star is normally taken as the Vaidya radiating metric with a null dust distribution. Maharaj et al. [32] proved that generalised exterior atmospheres arise in radiating stars in the form of the generalised Vaidya metric with a combination of null dust and a null string fluid. What is the general matter distribution in the interior of the radiating star that matches to such a matter combination (i.e. a generalised atmosphere) in the exterior? To answer this question we require that the interior and exterior matter distributions be physically relevant and the matching takes place across a comoving hypersurface. This is the objective of this paper. 
We find that our approach does lead to a result which is a generalisation of the Santos [1] boundary condition with a clear physical interpretation. Also previous results are regained as special cases.

The structure of the paper is as follows. In the following section we consider the interior spacetime with a composite matter distribution and derive the Einstein equations pertaining to this field. In Sect. 3 we consider the exterior spacetime to be the generalised Vaidya atmosphere and we derive the relevant field equations for this Type II fluid. In the following sections we demonstrate the matching conditions for the two spacetimes and note the differences contained in the boundary conditions we derived, compared to those of Santos $[1,32]$. These are then extended to higher dimensions in Sect. 5 where we demonstrate that the dimensionality of spacetime does not affect the final outcome of the stellar matching. Finally, in Sect. 6 we consider a shearing and anisotropic interior with a composite matter field and we obtain the boundary conditions for its matching to the generalised Vaidya metric. Concluding remarks are made in Sect. 7.

\section{Interior spacetime}

We take the interior spacetime $\mathscr{M}^{-}$to be the shear-free line element. Shear-free fluids are important in the modeling of inhomogeneous cosmological processes and radiating stars in relativistic astrophysics. Spherically symmetric spacetimes in the absence of shear can be written as

$d s_{-}^{2}=-A^{2} d t^{2}+B^{2}\left[d r^{2}+r^{2}\left(d \theta^{2}+\sin ^{2} \theta d \phi^{2}\right)\right]$,

in comoving coordinates $\left(x^{a}\right)=(t, r, \theta, \phi)$. The metric functions $A$ and $B$ depend on both the timelike coordinate $t$ and the radial coordinate $r$.

The nonvanishing Einstein tensor components are given by

$$
\begin{aligned}
G_{00}^{-}= & 3 \frac{\dot{B}^{2}}{B^{2}}-\frac{A^{2}}{B^{2}}\left(2 \frac{B^{\prime \prime}}{B}-\frac{B^{\prime 2}}{B^{2}}+\frac{4}{r} \frac{B^{\prime}}{B}\right), \\
G_{01}^{-}= & -\frac{2}{B^{2}}\left(B \dot{B}^{\prime}-B^{\prime} \dot{B}-B \dot{B} \frac{A^{\prime}}{A}\right), \\
G_{11}^{-}= & \frac{1}{A^{2}}\left(-2 B \ddot{B}-\dot{B}^{2}+2 B \dot{B} \frac{\dot{A}}{A}\right) \\
& +\frac{1}{B^{2}}\left(B^{\prime 2}+2 B B^{\prime} \frac{A^{\prime}}{A}\right. \\
& \left.+B^{2} \frac{2}{r} \frac{A^{\prime}}{A}+B B^{\prime} \frac{2}{r}\right),
\end{aligned}
$$

$$
\begin{aligned}
G_{22}^{-}= & -2 r^{2} \frac{B \ddot{B}}{A^{2}}+2 r^{2} B \dot{B} \frac{\dot{A}}{A^{3}}-r^{2} \frac{\dot{B}^{2}}{A^{2}} \\
& +r \frac{A^{\prime}}{A}+r \frac{B^{\prime}}{B}+r^{2} \frac{A^{\prime \prime}}{A} \\
& -r^{2} \frac{B^{\prime 2}}{B^{2}}+r^{2} \frac{B^{\prime \prime}}{B}, \\
G_{33}^{-}= & \sin ^{2} \theta G_{22}^{-},
\end{aligned}
$$

for the metric (1). In the above, primes denote differentiation with respect to the radial coordinate and dots represent time derivatives.

The energy momentum tensor is taken to be a generalised imperfect fluid of the form in the interior

$$
\begin{aligned}
T_{a b}^{-}= & (\rho+p) u_{a} u_{b}+p g_{a b}+q_{a} u_{b}+q_{b} u_{a} \\
& +\epsilon l_{a} l_{b}+(\mu+\mathscr{P})\left(l_{a} n_{b}+l_{b} n_{a}\right)+\mathscr{P} g_{a b} .
\end{aligned}
$$

In the above, $\rho$ is the energy density, $p$ is the isotropic pressure, $\mathbf{u}$ is the four-velocity, $\mathbf{q}$ is the heat flow vector, $\epsilon$ is the energy density of the internal null dust, $\mu$ is the null string energy density and $\mathscr{P}$ is the pressure of the internal null fluid. Since the unit timelike vector $\mathbf{u}$ is comoving we have that $u^{a} u_{a}=-1$ and $u^{a} q_{a}=0$ so that

$u^{a}=\frac{1}{A} \delta^{a}{ }_{0}, \quad q^{a}=\frac{1}{B} q \delta^{a}{ }_{1}$.

Note that the null vectors $\mathbf{l}$ and $\mathbf{n}$ satisfy

$l^{a} l_{a}=n^{a} n_{a}=0, \quad l_{a} n^{a}=-1$,

so that

$l^{a}=\frac{1}{A} \delta^{a}{ }_{0}+\frac{1}{B} \delta^{a}{ }_{1}$,

$n^{a}=\frac{1}{2 A} \delta^{a}{ }_{0}-\frac{1}{2 B} \delta^{a}{ }_{1}$.

The null vector $\mathbf{I}$ and the timelike vector $\mathbf{u}$ are related by

$l^{a} u_{a}=-1$.

The nonzero components of the energy momentum tensor (3) are then

$T_{00}^{-}=A^{2}(\rho+\epsilon+\mu)$,

$T_{01}^{-}=-A B(q+\epsilon)$,

$T_{11}^{-}=B^{2}(p+\epsilon-\mu)$,

$T_{22}^{-}=r^{2} B^{2}(p+\mathscr{P})$,

$T_{33}^{-}=\sin ^{2} \theta T_{22}$.

We observe that the matter distribution is a superposition of a barotropic fluid (with $\rho, p$ and $q$ ), null dust (with $\epsilon$ ) and a null string fluid (with $\mu$ and $\mathscr{P}$ ). The treatment of Di Prisco et al. [15] does not contain the null string distribution for the stellar interior.

The null string fluid also plays a significant role in the process of gravitational collapse including diffusive transport as shown by Husain [33] and Glass and Krisch [34,35]. As far 
as we are aware, the combination of the sources (barotropic fluid, null dust and null string fluid) in the energy momentum (3) and (9) has not been considered before in the context of a radiating star.

The Einstein field equations $G_{a b}^{-}=T_{a b}^{-}$are then given by

$$
\begin{aligned}
\rho+\epsilon+\mu= & \frac{3 \dot{B}^{2}}{A^{2} B^{2}} \\
& -\frac{1}{B^{2}}\left(\frac{2 B^{\prime \prime}}{B}-\frac{B^{\prime 2}}{B^{2}}+\frac{4 B^{\prime}}{r B}\right), \\
p+\epsilon-\mu= & \frac{1}{A^{2}}\left(\frac{-2 \ddot{B}}{B}-\frac{\dot{B}^{2}}{B^{2}}+\frac{2 \dot{A} \dot{B}}{A B}\right) \\
& +\frac{1}{B^{2}}\left(\frac{B^{\prime 2}}{B^{2}}+\frac{2 A^{\prime} B^{\prime}}{A B}+\frac{2 A^{\prime}}{r A}+\frac{2 B^{\prime}}{r B}\right), \\
p+\mathscr{P}= & -\frac{2 \ddot{B}}{B A^{2}}+\frac{2 \dot{A} \dot{B}}{B A^{3}}-\frac{\dot{B}^{2}}{A^{2} B^{2}}+\frac{A^{\prime}}{r A B^{2}} \\
& +\frac{B^{\prime}}{r B^{3}}+\frac{A^{\prime \prime}}{A B^{2}}-\frac{B^{\prime 2}}{B^{4}}+\frac{B^{\prime \prime}}{B^{3}}, \\
q+\epsilon= & -\frac{2}{A B}\left(-\frac{\dot{B}^{\prime}}{B}+\frac{B^{\prime} \dot{B}}{B^{2}}+\frac{A^{\prime}}{A} \frac{\dot{B}}{B}\right),
\end{aligned}
$$

for the shear-free metric. The field equations (10) are a system of coupled and highly nonlinear partial differential equations that describe the dynamics of the generalised matter field in the interior of the radiating star. In the above, the physical quantities $\rho, p, q, \epsilon, \mu$ and $\mathscr{P}$ all depend on the coordinates $t$ and $r$. An interesting note here is if the heat flux $q$ vanishes in equation (10d), then there is an explicit expression for the internal null energy density $\epsilon$ which is a direct consequence of the generalised matter distribution (3).

\section{Exterior spacetime}

The spacetime outside a spherically symmetric radiating star is described by the Vaidya geometry and it defines outgoing null radiation. The metric is written in terms of the mass of the radiating body and its Petrov-Pirani-Penrose classification is type D [36]. The generalisation of the Vaidya spacetime was given in detail in [37-39] and includes most of the known solutions of Einstein's field equations with matter distributions of a Type II fluid. For the exterior spacetime manifold $\mathscr{M}^{+}$, the generalised Vaidya metric with (exploding) null coordinates $(v, \boldsymbol{r}, \theta, \phi)$ is given as

$$
\begin{aligned}
d s_{+}^{2}= & -\left(1-\frac{2 m(v, \mathrm{r})}{\mathrm{r}}\right) d v^{2} \\
& -2 d v d \mathrm{r}+\mathrm{r}^{2}\left(d \theta^{2}+\sin ^{2} \theta d \phi^{2}\right) .
\end{aligned}
$$

Here the function $m(v, r)$ describes the above mentioned Misner-Sharp mass of the star, which is also called the mass function $[40,41]$. It gives a measure of the gravitational energy within a given radius $r$.

The nonvanishing components of the Einstein tensor are given by

$G_{00}^{+}=-\frac{2}{\mathrm{r}^{3}}\left(2 m m_{\mathrm{r}}-m_{\mathrm{r}}+\mathrm{r} m_{v}\right)$,

$G_{01}^{+}=\frac{2 m_{\mathrm{r}}}{\mathrm{r}^{2}}$,

$G_{22}^{+}=-r m_{\mathrm{rr}}$,

$G_{33}^{+}=\sin ^{2} \theta G_{22}^{+}$,

where we have used $m_{v}=\frac{\partial m}{\partial v}$ and $m_{\mathrm{r}}=\frac{\partial m}{\partial \mathrm{r}}$. The exterior energy momentum tensor is defined by

$T_{a b}^{+}=T_{a b}^{(n)}+T_{a b}^{(m)}$,

which is a superposition of null dust and null string fluids. We can write

$T_{a b}^{+}=\bar{\epsilon} \bar{l}_{a} \bar{l}_{b}+(\bar{\mu}+\overline{\mathscr{P}})\left(\bar{l}_{a} \bar{n}_{b}+\bar{l}_{b} \bar{n}_{a}\right)+\overline{\mathscr{P}}_{g_{a b}}$.

In the above we have

$\bar{l}_{a}=\delta^{0}{ }_{a}, \quad \bar{n}_{a}=\frac{1}{2}\left[1-\frac{2 m(v, \mathrm{r})}{\mathrm{r}}\right] \delta^{0}{ }_{a}+\delta^{1}{ }_{a}$,

with $\bar{l}_{c} \bar{l}^{c}=\bar{n}_{c} \bar{n}^{c}=0$ and $\bar{l}_{c} \bar{n}^{c}=-1$. The null vector $\bar{l}^{a}$ is a double null eigenvector of the energy momentum tensor (13). The nonzero components of (13) are

$T_{00}^{+}=\bar{\epsilon}+\bar{\mu}\left(1-\frac{2 m}{r}\right)$,

$T_{01}^{+}=\bar{\mu}$,

$T_{22}^{+}=\mathrm{r}^{2} \overline{\mathscr{P}}$

$T_{33}^{+}=\sin ^{2} \theta T_{22}^{+}$.

In general, $T_{a b}^{+}$represents a Type II fluid (see Hawking and Ellis [42]).

The Einstein field equations $G_{a b}^{+}=T_{a b}^{+}$for the exterior spacetime manifold $\mathscr{M}^{+}$are then given by

$$
\begin{aligned}
\bar{\varepsilon} & =-\frac{2 m_{v}}{\mathrm{r}^{2}}, \\
\bar{\mu} & =\frac{2 m_{\mathrm{r}}}{\mathrm{r}^{2}}, \\
\overline{\mathscr{P}} & =-\frac{m_{\mathrm{rr}}}{\mathrm{r}} .
\end{aligned}
$$

In the field equations (16), $\bar{\varepsilon}$ is the energy density of the null dust radiation, $\bar{\mu}$ is the null string energy density and $\bar{P}$ is the null string pressure. These are defined in the external atmosphere of the star. They depend on the coordinates $v$ and $r$. 


\section{Matching of the two spacetimes}

The model for a relativistic, dissipating radiating star was completed by Santos [1] by analysing the junction conditions at the stellar surface. The important result that followed was that the pressure on the boundary of the radiating star should be nonzero in general, and proportional to the heat flux. This result was further generalised by Maharaj et al. [32] for an exterior Type II fluid. The matching of the interior manifold $\mathscr{M}^{-}$to the exterior manifold $\mathscr{M}^{+}$across the hypersurface $\Sigma$ is studied. We provide only the essential steps to highlight the role played by the null quantities $\epsilon, \mu$ and $\bar{\mu}$, normally absent in other investigations. The background theoretical details of the junction conditions are provided in the Appendix.

The intrinsic metric to $\Sigma$ is given by

$d s_{\Sigma}^{2}=-d \tau^{2}+\mathscr{R}^{2}\left(d \theta^{2}+\sin ^{2} \theta d \phi^{2}\right)$,

where $\mathscr{R}=\mathscr{R}(\tau)$ and coordinates $\xi^{i}=(\tau, \theta, \phi)$. It is important to note that the coordinate $\tau$ is defined only on $\Sigma$. The surface $\Sigma$ is the boundary of the interior distribution of matter (1) and in this case is given by

$f(r, t)=r-r_{\Sigma}=0$,

where $r_{\Sigma}$ is a constant. The vector orthogonal to $\Sigma$ is $\frac{\partial f}{\partial \chi_{-}^{a}}$ and is given by

$\frac{\partial f}{\partial \chi_{-}^{a}}=(0,1,0,0)$,

so that the unit vector normal to the surface $\Sigma$ takes the form

$n_{a}^{-}=\left[0, B\left(r_{\Sigma}, t\right), 0,0\right]$.

For the interior manifold $\mathscr{M}^{-}$the first junction condition (A.4), for the line elements (1) and (17), yields the following restrictions

$$
A\left(r_{\Sigma}, t\right) \grave{t}=1
$$

$r_{\Sigma} B\left(r_{\Sigma}, t\right)=\mathscr{R}(\tau)$,

where $\grave{t}=\frac{d t}{d \tau}$. The extrinsic curvature components $K_{i j}^{-}$of $\Sigma$ can be obtained by using (A.6), (1) and (18) yielding

$K_{\tau \tau}^{-}=\left(-\frac{1}{B} \frac{A^{\prime}}{A}\right)_{\Sigma}$,

$K_{\theta \theta}^{-}=\left(r(r B)^{\prime}\right)_{\Sigma}$,

$K_{\phi \phi}^{-}=\sin ^{2} \theta K_{\theta \theta}^{-}$,

which are valid on the surface $\Sigma$.

For the exterior spacetime $\mathscr{M}^{+}$, the equation of the surface $\Sigma$ is given by

$f(\mathrm{r}, v)=\mathrm{r}-\mathrm{r}_{\Sigma}=0$

hence the vector orthogonal to $\Sigma$ is $\frac{\partial f}{\partial \chi_{+}^{a}}=\left(-\frac{d r_{\Sigma}}{d v}, 1,0,0\right)$.

The unit normal vector is then

$n_{a}^{+}=\left(1-\frac{2 m}{\mathrm{r}_{\Sigma}}+2 \frac{d \mathrm{r}_{\Sigma}}{d v}\right)^{-\frac{1}{2}}\left(-\frac{d \mathrm{r}_{\Sigma}}{d v}, 1,0,0\right)$,

on $\Sigma$. The first junction condition (A.4) for the spacetimes (11) and (17) gives the following

$$
\begin{aligned}
& \mathrm{r}_{\Sigma}=\mathscr{R}(\tau), \\
& \left(1-\frac{2 m}{\mathrm{r}}+2 \frac{d \mathrm{r}}{d v}\right)_{\Sigma}=\left(\frac{1}{\bar{v}^{2}}\right)_{\Sigma} .
\end{aligned}
$$

The unit normal vector (21) can be rewritten, using (22b) in the following simpler form

$n_{a}^{+}=(-\grave{r}, \grave{v}, 0,0)$.

Using (A.6), (11) and the above Eq. (23), we can calculate the nonvanishing extrinsic curvature components given by

$K_{\tau \tau}^{+}=\left(\frac{\grave{i}}{\grave{v}}-\grave{v} \frac{m}{\mathrm{r}^{2}}+\grave{v} \frac{m_{\mathrm{r}}}{\mathrm{r}}\right)_{\Sigma}$

$K_{\theta \theta}^{+}=\left(\grave{v}\left(1-\frac{2 m}{\mathrm{r}}\right)-\mathrm{rr}\right)_{\Sigma}$,

$K_{\phi \phi}^{+}=\sin ^{2} \theta K_{\theta \theta}^{+}$,

which are valid on the surface $\Sigma$. The first junction condition (A.4) yields the equations (19) and (22), which are summarised below

$$
\begin{aligned}
& A\left(r_{\Sigma}, t\right) \grave{t}=1, \\
& r_{\Sigma} B\left(r_{\Sigma}, t\right)=\mathscr{R}(\tau), \\
& r_{\Sigma}(v)=\mathscr{R}(\tau), \\
& \left(1-\frac{2 m}{\mathrm{r}}+2 \frac{d \mathrm{r}}{d v}\right)_{\Sigma}=\left(\frac{1}{\grave{v}^{2}}\right)_{\Sigma} .
\end{aligned}
$$

Since the variable $\tau$ was only defined as an intermediate variable, it can be eliminated from the above equations. Thus, the necessary and sufficient conditions on the spacetimes for the validity of the first junction condition (A.4) are

$$
\begin{aligned}
(A d t)_{\Sigma} & =\left(1-\frac{2 m}{\mathrm{r}}+2 \frac{d \mathrm{r}}{d v}\right)_{\Sigma}^{\frac{1}{2}}, \\
(r B)_{\Sigma} & =\mathrm{r}_{\Sigma}(v) .
\end{aligned}
$$

Equating the appropriate extrinsic curvature components (20) and (24), yields the second junction condition (A.5) as

$$
\begin{aligned}
\left(-\frac{1}{B} \frac{A^{\prime}}{A}\right)_{\Sigma} & =\left(\frac{\grave{v}}{\grave{v}}-\grave{v} \frac{m}{\mathrm{r}^{2}}+\grave{v} \frac{m_{\mathrm{r}}}{\mathrm{r}}\right)_{\Sigma}, \\
\left(r(r B)^{\prime}\right)_{\Sigma} & =\left(\grave{v}\left(1-\frac{2 m}{\mathrm{r}}\right)-\mathrm{rr}\right)_{\Sigma} .
\end{aligned}
$$

We can obtain an expression for $m(v)$ in terms of $A$ and $B$ only by eliminating $r, \grave{r}$ and $\grave{v}$ from Eq. (27b) above. The mass 
function can be written after a long calculation, with the aid of (25), as

$m(v, r)=\left[\frac{r B}{2}\left(1+r^{2} \frac{\dot{B}^{2}}{A^{2}}-\frac{1}{B^{2}}\left(B+r B^{\prime}\right)\right)\right]_{\Sigma}$.

The expression above is interpreted as the total gravitational mass of the star within the surface $\Sigma$. From Eqs. (25a), (25b) and $(25 \mathrm{c})$ we have

$\grave{\mathrm{r}}_{\Sigma}=\left(\frac{r \dot{B}}{A}\right)_{\Sigma}$,

and substituting the mass function (28) into (27b), using the expression for $\dot{\mathrm{r}}_{\Sigma}$ above, we get

$\grave{v}_{\Sigma}=\left(1+r \frac{B^{\prime}}{B}+r \frac{\dot{B}}{A}\right)_{\Sigma}^{-1}$.

Differentiating the above expression with respect to $\tau$ and using (25a), we acquire

$$
\begin{aligned}
\grave{\grave{v}}_{\Sigma}= & {\left[\frac{1}{A}\left(1+r \frac{B^{\prime}}{B}+r \frac{\dot{B}}{A}\right)^{-2}\right.} \\
& \left.\times\left(r \frac{\dot{B} B^{\prime}}{B^{2}}-r \frac{\dot{B}^{\prime}}{B}+r \frac{\dot{A} \dot{B}}{A}-r \frac{\ddot{B}}{B}\right)\right]_{\Sigma} .
\end{aligned}
$$

Upon substituting (28), (29) and (30) into (27a), we obtain after some lengthy algebra

$$
\begin{aligned}
& 2 \frac{\dot{B}^{\prime}}{A B^{2}}-2 \frac{B^{\prime} \dot{B}}{A B^{3}}-2 \frac{A^{\prime}}{A^{2}} \frac{\dot{B}}{B^{2}}=2 \frac{m_{\mathrm{r}}}{\mathrm{r}^{2}}+\frac{1}{A^{2}}\left(\frac{-2 \ddot{B}}{B} \frac{\dot{B}^{2}}{B^{2}}+\frac{2 \dot{A} \dot{B}}{A B}\right) \\
& +\frac{1}{B^{2}}\left(\frac{B^{\prime 2}}{B^{2}}+\frac{2 A^{\prime} B^{\prime}}{A B}+\frac{2 A^{\prime}}{r A}+\frac{2 B^{\prime}}{r B}\right) .
\end{aligned}
$$

Simplifying the above, using the field equations (10b) and (10d), we acquire the result

$(p+\epsilon-\mu)_{\Sigma}=\left(q+\epsilon-2 \frac{m_{\mathrm{r}}}{\mathrm{r}^{2}}\right)_{\Sigma}$.

There are three interesting features in equation (32). Firstly, the appearance of the term containing $m_{\mathrm{r}}$, a new contribution from the Vaidya mass function, which does not appear in the treatment of Santos [1]. A similar observation of this feature was made in [32]. Secondly, the left hand side of the equation (32) corresponds to $p+\epsilon-\mu$ which is the same as the field equation (10b). Thirdly, the right hand side of (32) contains the term $q+\epsilon$ which is equivalent to the field equation (10d). We then have that (32) takes the remarkably simple form

$p_{\Sigma}=\left(q+\mu-2 \frac{m_{\mathrm{r}}}{\mathrm{r}^{2}}\right)_{\Sigma}$.

We observe from (16b) that the term $\frac{2 m_{\mathrm{r}}}{r^{2}}$ is the external null string density which is an added contribution to the junction condition. Hence we can write the said condition at the stellar surface $\Sigma$ in the transparent form

$p_{\Sigma}=(q+\mu-\bar{\mu})_{\Sigma}$, which is our main result. We have established the general junction condition (34) for the interior shear-free matter distribution (3) in $\mathscr{M}^{-}$and the exterior generalised Vaidya atmosphere with the matter distribution (15) in $\mathscr{M}^{+}$.

From (34) we observe that the isotropic pressure $p$, the heat flux $q$, the internal string density $\mu$ and the external string density $\bar{\mu}$ determine the dynamical evolution of a radiating star with outgoing dissipation in the form of a radial heat flow. The pressure on the stellar surface depends on the difference $\mu-\bar{\mu}$ of the null string densities from $\mathscr{M}^{-}$and $\mathscr{M}^{+}$, respectively. The important physical observation that can be made from our analysis is that the internal null string density $\mu$ directly affects the pressure $p$ at the boundary $\Sigma$. This physical feature is absent in the treatment of Santos [1] and Maharaj et al. [32]. If $\mu-\bar{\mu}>0$ then we obtain higher values for the pressure on $\Sigma$. If $\mu-\bar{\mu}<0$ then we obtain lower values for the pressure on the boundary. This difference $\mu-\bar{\mu}$ affects the evolution of the radiating star in general relativity, as well as the rate of gravitational collapse, with $\mu-\bar{\mu} \gtrless 0$ leading to greater/lower pressures at the stellar surface during the collapse process. Further to these notions, the new result is indeed extensive and may serve as being applicable to physically significant astrophysical frameworks as the effects of the null fluid string energy densities $\mu$ and $\bar{\mu}$ cannot be overlooked. This result may also become appropriate for the description of neutrino outflows from highly compact relativistic stars, post collapse, in which the nonadiabatic and particle production processes reign in the strong gravity regime [43]. In light of this, it should be noted that this generalised model gives significant improvement on the approaches found in $[1,32,44]$.

A number of previous results are contained in our treatment. When $q=\mu=\bar{\mu}=0$, then $p=0$ on $\Sigma$ and we obtain the Schwarzschild exterior geometry. If $\mu=\bar{\mu}=0$, then (34) gives $p=q$ on $\Sigma$, regaining the familiar Santos [1] junction condition. For the case $\mu=0$, the boundary condition yields $p=q-\bar{\mu}$ on $\Sigma$ which was first found by Maharaj et al. [32]. Note from (34) that when $q=\mu-\bar{\mu}$, then $p=0$ on $\Sigma$ and the exterior geometry is still described by the generalised Vaidya spacetime. In addition, when $q=0$, we then have that $p=\mu-\bar{\mu}$ on $\Sigma$ and the interior spacetime $\mathscr{M}^{-}$does not radiate. In this case, the isotropic pressure is sustained on the boundary $\Sigma$ by the null string densities $\mu$ and $\bar{\mu}$.

\section{Extension to arbitrary dimensions}

The calculation of the junction conditions in higher dimensions was done by Bhui et al. [45], Banerjee et al. [46] and Shah et al. [47] where it was shown that the dimensionality of the spacetime does not materially alter the results of the usual 
four-dimensional counterpart. In this section, we will show that the same notion holds for our generalised distributions.

For the interior, we take the $N$-dimensional shear-free spacetime metric as

$d s_{-}^{2}=-A^{2} d t^{2}+B^{2}\left[d r^{2}+r^{2} d \Omega_{N-2}^{2}\right]$,

where we have the $(N-2)$-sphere

$d \Omega_{N-2}^{2}=\sum_{i=1}^{N-2}\left[\prod_{j=1}^{i-1} \sin ^{2}\left(\theta_{j}\right)\right]\left(d \theta_{i}\right)^{2}$.

The resulting Einstein field equations are thus given by

$$
\begin{aligned}
\rho+\epsilon+\mu= & \frac{(N-1)(N-2)}{2} \frac{\dot{B}^{2}}{A^{2} B^{2}}-\frac{(N-2)}{B^{2}}\left[\frac{B^{\prime \prime}}{B}\right. \\
& \left.+\left(\frac{N-5}{2}\right) \frac{B^{\prime 2}}{B^{2}}+(N-2) \frac{B^{\prime}}{r B}\right], \\
p+\epsilon-\mu= & \frac{(N-2)}{A^{2}} \\
& \times\left[-\frac{\ddot{B}}{B}-\left(\frac{N-3}{2}\right) \frac{\dot{B}^{2}}{B^{2}}+\frac{\dot{A} \dot{B}}{A B}\right] \\
& +\frac{(N-2)}{B^{2}} \\
& \times\left[\left(\frac{N-3}{2}\right) \frac{B^{\prime 2}}{B^{2}}+\frac{A^{\prime} B^{\prime}}{A B}+\frac{A^{\prime}}{r A}+(N-3) \frac{B^{\prime}}{r B}\right], \\
p+\mathscr{P}= & \frac{1}{B^{2}}\left[\frac{A^{\prime \prime}}{A}+(N-3) \frac{B^{\prime \prime}}{B}+(N-3) \frac{A^{\prime}}{r A}\right. \\
& +\left(\frac{(N-3)(N-6)}{2}\right) \frac{B^{\prime 2}}{B^{2}} \\
& \left.+(N-3)^{2} \frac{B^{\prime}}{r B}+(N-4) \frac{A^{\prime} B^{\prime}}{A B}\right]-\frac{(N-2)}{A^{2}}\left[\frac{\ddot{B}}{B}\right. \\
& \left.+\left(\frac{N-3}{2}\right) \frac{\dot{B}^{2}}{B^{2}}-\frac{\dot{A} \dot{B}}{A B}\right], \\
q+\epsilon= & \frac{(N-2)}{A B} \\
& {\left[-\frac{\dot{B}^{\prime}}{B}+\frac{B^{\prime} \dot{B}}{B^{2}}+\frac{A^{\prime}}{A} \frac{\dot{B}}{B}\right], } \\
& {\left[\left(\frac{N}{A}\right]\right.}
\end{aligned}
$$

for the spacetime (35).

For the exterior spacetime, we take the generalised Vaidya metric in $N$ dimensions

$$
\begin{aligned}
d s_{+}^{2}= & -\left(1-\frac{2 m(v, \mathrm{r})}{(N-3) \mathrm{r}^{N-3}}\right) d v^{2} \\
& -2 d v d \mathrm{r}+\mathrm{r}^{2} d \Omega_{N-2}^{2},
\end{aligned}
$$

where we have the $(N-2)$-sphere $d \Omega_{N-2}^{2}$ as before.

The resulting field equations are therefore

$$
\begin{aligned}
\bar{\epsilon} & =-\frac{(N-2) m_{v}}{\mathrm{r}^{N-2}}, \\
\bar{\mu} & =\frac{(N-2) m_{\mathrm{r}}}{\mathrm{r}^{N-2}}, \\
\overline{\mathscr{P}} & =-\frac{m_{\mathrm{rr}}}{\mathrm{r}^{N-3}},
\end{aligned}
$$

for the metric (37). When $N=4$ we regain the fourdimensional field equations (16).

The $(N-1)$-dimensional intrinsic metric to $\Sigma$ is given by

$d s_{\Sigma}^{2}=-d \tau^{2}+\mathscr{R}^{2} d \Omega_{N-2}^{2}$,

where $\mathscr{R}=\mathscr{R}(\tau)$ and we have coordinates

$\xi^{i}=\left(\tau, \theta_{1}, \theta_{2}, \ldots, \theta_{N-2}\right)$.

The surface $\Sigma$ is the boundary of the interior distribution of matter (35) and is given by

$f(r, t)=r-r_{\Sigma}=0$,

where $r_{\Sigma}$ is a constant. The $N$-dimensional vector orthogonal to $\Sigma$ is $\frac{\partial f}{\partial \chi_{-}^{a}}$ and is given by

$\frac{\partial f}{\partial \chi_{-}^{a}}=(0,1,0,0, \ldots, 0)$,

therefore the unit vector normal to the surface $\Sigma$ takes the form

$n_{a}^{-}=\left[0, B\left(r_{\Sigma}, t\right), 0,0,0, \ldots, 0\right]$.

The extrinsic curvature components $K_{i j}^{-}$of $\Sigma$ for the interior spacetime are calculated to be

$$
\begin{aligned}
K_{\tau \tau}^{-} & =\left(-\frac{1}{B} \frac{A^{\prime}}{A}\right)_{\Sigma}, \\
K_{\theta_{1} \theta_{1}}^{-} & =\left(r(r B)^{\prime}\right)_{\Sigma}, \\
K_{\theta_{N-2} \theta_{N-2}}^{-} & =\left[\prod_{j=1}^{N-2} \sin ^{2}\left(\theta_{j}\right)\right] K_{\theta_{1} \theta_{1}}^{-} .
\end{aligned}
$$

For the exterior Vaidya spacetime, the equation of the surface $\Sigma$ is given by

$f(\mathrm{r}, v)=\mathrm{r}-\mathrm{r}_{\Sigma}=0$,

hence the vector orthogonal to $\Sigma$ is

$\frac{\partial f}{\partial \chi_{+}^{a}}=\left(-\frac{d \mathrm{r}_{\Sigma}}{d v}, 1,0,0, \ldots, 0\right)$.

The unit normal vector is then

$$
\begin{aligned}
n_{a}^{+}= & \left(1-\frac{2 m}{(N-3) \mathrm{r}_{\Sigma}^{N-3}}+2 \frac{d \mathrm{r}_{\Sigma}}{d v}\right)^{-\frac{1}{2}} \\
& \times\left(-\frac{d \mathrm{r}_{\Sigma}}{d v}, 1,0,0, \ldots, 0\right),
\end{aligned}
$$

on $\Sigma$. 
The extrinsic curvature components $K_{i j}^{+}$of $\Sigma$ are given by

$$
\begin{aligned}
K_{\tau \tau}^{+} & =\left(\frac{\grave{v}}{\grave{v}}-\grave{v} \frac{m}{\mathrm{r}^{N-2}}+\grave{v} \frac{m_{\mathrm{r}}}{\mathrm{r}^{N-3}}\right)_{\Sigma}, \\
K_{\theta_{1} \theta_{1}}^{+} & =\left(\grave{v}\left(1-\frac{2 m \mathrm{r}^{3-N}}{(N-3)}\right)-\grave{\mathrm{r}}\right)_{\Sigma}, \\
K_{\theta_{N-2} \theta_{N-2}}^{+} & =\left[\prod_{j=1}^{N-2} \sin ^{2}\left(\theta_{j}\right)\right] K_{\theta_{1} \theta_{1}}^{+} .
\end{aligned}
$$

Thus, the necessary and sufficient conditions on the spacetimes for the validity of the first junction condition (A.4) are

$$
\begin{aligned}
(A d t)_{\Sigma} & =\left(1-\frac{2 m}{(N-3) r^{N-3}}+2 \frac{d r}{d v}\right)_{\Sigma}^{\frac{1}{2}} \\
(r B)_{\Sigma} & =r_{\Sigma}(v) .
\end{aligned}
$$

Equating the appropriate extrinsic curvature components (41) and (43), yields the second junction condition (A.5) as

$$
\begin{aligned}
\left(-\frac{1}{B} \frac{A^{\prime}}{A}\right)_{\Sigma} & =\left(\frac{\grave{v}}{\grave{v}}-\grave{v} \frac{m}{\mathrm{r}^{N-2}}+\grave{v} \frac{m_{\mathrm{r}}}{\mathrm{r}^{N-3}}\right)_{\Sigma}, \\
\left(r(r B)^{\prime}\right)_{\Sigma} & =\left(\grave{v}\left(1-\frac{2 m \mathrm{r}^{3-N}}{(N-3)}\right)-\mathrm{rr}\right)_{\Sigma} .
\end{aligned}
$$

Following the same methodology as in the previous section, we find after a lengthy calculation that the mass function is given by

$$
\begin{aligned}
m(v, r)= & (N-3)(r B)^{N-3} \\
& \times\left(r^{2} \frac{\dot{B}^{2}}{2 A^{2}}-r \frac{B^{\prime}}{B}-r^{2} \frac{B^{\prime 2}}{2 B^{2}}\right),
\end{aligned}
$$

which is expressed only in terms of the interior potentials. Using the gravitational mass function in the second junction condition (45a) yields

$$
\begin{aligned}
(N-2)\left[-\frac{\dot{B}^{\prime}}{A B^{2}}+\frac{B^{\prime} \dot{B}}{A B^{3}}+\frac{A^{\prime}}{A^{2}} \frac{\dot{B}}{B^{2}}\right] \\
=\frac{(N-2) m_{\mathrm{r}}}{\mathrm{r}^{N-2}+\frac{(N-2)}{A^{2}}\left[-\frac{\ddot{B}}{B}\right.} \\
\left.\quad-\left(\frac{N-3}{2}\right) \frac{\dot{B}^{2}}{B^{2}}+\frac{\dot{A} \dot{B}}{A B}\right] \\
+\frac{(N-2)}{B^{2}}\left[\left(\frac{N-3}{2}\right) \frac{B^{\prime 2}}{B^{2}}+\frac{A^{\prime} B^{\prime}}{A B}+\frac{A^{\prime}}{r A}+(N-3) \frac{B^{\prime}}{r B}\right] .
\end{aligned}
$$

Equation (47) is the higher dimensional generalisation of (31), to which it reduces when $N=4$. This emphasises the role of the dimension $N$ in the collapse process. This feature is also illustrated in the work of Mkenyeleye et al. [48] who showed that naked singularities become covered in higher dimensions. We can show that (47) reduces to the simpler form

$p_{\Sigma}=(q+\mu-\bar{\mu})_{\Sigma}$,

where we have utilised the field equations (36b), (36d) and (38b). It can be seen that the boundary condition (48) is analogous to its four-dimensional counterpart (34), and therefore holds in any dimension greater than four. Note that the dimension $N$ is contained implicitly in the junction condition through the pressure $p$ and heat flux $q$ via equations (36b) and (36d) respectively.

\section{Junction conditions with shear and anisotropic stresses}

The junction conditions of Santos [1] have been generalised to include shear, electromagnetic field, bulk viscosity and anisotropic stresses [5,49,50]. These models have proved efficacious as resources for physically viable and tractable stellar models describing acceleration-free collapse, contraction from an initial static configuration, expansion-free collapse, gamma-ray bursts and Euclidean stars. Such physical systems have been analysed within the framework of extended irreversible thermodynamics to generate temperature profiles for ultra relativistic particles. It is therefore important to consider radiating structures with the generalised matter distribution (3), extended to include anisotropic matter, for a general spherically symmetric metric with shear.

In this section, we will present the generalised junction conditions for a radiating star with nonvanishing shearing stresses. We will consider the interior spacetime to now be the general shearing metric, given by

$d s_{-}^{2}=-A^{2} d t^{2}+B^{2} d r^{2}+Y^{2}\left(d \theta^{2}+\sin ^{2} \theta d \phi^{2}\right)$,

where the metric functions $A=A(r, t), B=B(r, t)$ and $Y=Y(r, t)$. The fluid four-velocity is comoving as in the previous case and so $u^{a}=\frac{1}{A} \delta^{a} 0$. The kinematical quantities are

$$
\begin{aligned}
\omega_{a b} & =0, \\
\dot{u}^{a} & =\frac{A^{\prime}}{A} \delta^{a}{ }_{1}, \\
\Theta & =\frac{1}{A}\left(\frac{\dot{B}}{B}+2 \frac{\dot{Y}}{Y}\right), \\
\sigma^{1}{ }_{1} & =\sigma^{2}{ }_{2}=-\frac{1}{2} \sigma^{3}{ }_{3}=\frac{1}{\sqrt{3} A}\left(\frac{\dot{Y}}{Y}-\frac{\dot{B}}{B}\right),
\end{aligned}
$$

where $\omega_{a b}$ is the vorticity tensor, $\dot{u}^{a}$ is the fluid fouracceleration, $\Theta$ is the expansion invariant and $\sigma$ is the magnitude of the shear. The shear-free line element (1) can be regained when 
$\frac{\dot{Y}}{Y}-\frac{\dot{B}}{B}=0$,

using (50d).

The full energy momentum tensor for the above spacetime is written as

$$
\begin{aligned}
T_{a b}^{-}= & (\rho+p) u_{a} u_{b}+p g_{a b}+q_{a} u_{b}+q_{b} u_{a} \\
& +\epsilon l_{a} l_{b}+(\mu+\mathscr{P})\left(l_{a} n_{b}+l_{b} n_{a}\right) \\
& +\mathscr{P} g_{a b}+\pi_{a b},
\end{aligned}
$$

where the additional tensorial function $\pi_{a b}$ is the anisotropic stress tensor defined as

$\pi_{a b}=\Delta\left(\mathrm{n}_{a} \mathrm{n}_{b}-\frac{1}{3} h_{a b}\right)$.

In the above, the quantity $\Delta=p_{\|}-p_{\perp}$ is the degree of anisotropy. We have that $p_{\|}$is the radial pressure, $p_{\perp}$ is the tangential pressure and $\mathrm{n}_{a}$ is a unit radial vector defined by

$\mathrm{n}^{a}=\frac{1}{B} \delta^{a}$

The quantity $h_{a b}=g_{a b}+u_{a} u_{b}$ is the projection tensor. The isotropic pressure

$p=\frac{1}{3}\left(p_{\|}+2 p_{\perp}\right)$,

relates the radial pressure and the tangential pressure. The nonzero components of (51) are thus given by

$T_{00}^{-}=A^{2}(\rho+\epsilon-\mu)$,

$T_{01}^{-}=-A B(q+\epsilon)$,

$T_{11}^{-}=B^{2}\left(p+\epsilon-\mu+\frac{2}{3} \Delta\right)$,

$T_{22}^{-}=Y^{2}\left(p+\mathscr{P}-\frac{1}{3} \Delta\right)$,

$T_{33}^{-}=\sin ^{2} \theta T_{22}$,

where we have utilised (52). We regain the isotropic pressure $p=p_{\|}=p_{\perp}$, when $\Delta=0$.
The Einstein field equations, with shear and anisotropic pressures, are therefore

$$
\begin{aligned}
& \rho+\epsilon-\mu=\frac{2}{A^{2}} \frac{\dot{B} \dot{Y}}{B Y}+\frac{1}{Y^{2}}+\frac{1}{A^{2}} \frac{\dot{Y}^{2}}{Y^{2}} \\
& -\frac{1}{B^{2}}\left(2 \frac{Y^{\prime \prime}}{Y}+\frac{Y^{\prime 2}}{Y^{2}}-2 \frac{B^{\prime} Y^{\prime}}{B Y}\right) \\
& p+\epsilon-\mu+\frac{2}{3} \Delta=\frac{1}{A^{2}}\left(-2 \frac{\ddot{Y}}{Y}-\frac{\dot{Y}^{2}}{Y^{2}}\right. \\
& \left.+2 \frac{\dot{A} \dot{Y}}{A Y}\right)+\frac{1}{B^{2}}\left(\frac{Y^{\prime 2}}{Y^{2}}+2 \frac{A^{\prime} Y^{\prime}}{A Y}\right)-\frac{1}{Y^{2}}, \\
& p+\mathscr{P}-\frac{1}{3} \Delta=-\frac{1}{A^{2}}\left(\frac{\ddot{B}}{B}-\frac{\dot{A} \dot{B}}{A B}+\frac{\dot{B} \dot{Y}}{B Y}-\frac{\dot{A} \dot{Y}}{A Y}+\frac{\ddot{Y}}{Y}\right) \\
& +\frac{1}{B^{2}}\left(\frac{A^{\prime \prime}}{A}-\frac{A^{\prime} B^{\prime}}{A B}+\frac{A^{\prime} Y^{\prime}}{A Y}-\frac{B^{\prime} Y^{\prime}}{B Y}+\frac{Y^{\prime \prime}}{Y}\right), \\
& q+\epsilon=-\frac{2}{A B}\left(-\frac{\dot{Y}^{\prime}}{Y}+\frac{\dot{B} Y^{\prime}}{B Y}+\frac{A^{\prime} \dot{Y}}{A Y}\right)
\end{aligned}
$$

for the general spherically symmetric metric (49).

The exterior spacetime will again be the generalised Vaidya radiating metric

$$
\begin{aligned}
d s_{+}^{2}= & -\left(1-\frac{2 m(v, \mathrm{r})}{\mathrm{r}}\right) d v^{2} \\
& -2 d v d \mathrm{r}+\mathrm{r}^{2}\left(d \theta^{2}+\sin ^{2} \theta d \phi^{2}\right) .
\end{aligned}
$$

Utilising the same approach as that given in Sect. 4 the necessary and sufficient conditions for the validity of the first junction condition (A.4) on the two spacetimes is

$$
\begin{aligned}
(A d t)_{\Sigma} & =\left(1-\frac{2 m}{\mathrm{r}}+2 \frac{d \mathrm{r}}{d v}\right)_{\Sigma}^{\frac{1}{2}} d v \\
Y_{\Sigma} & =\mathrm{r}_{\Sigma},
\end{aligned}
$$

The second junction condition (A.5) gives the following necessary and sufficient conditions on the two spacetime manifolds

$$
\begin{aligned}
\left(-\frac{1}{B} \frac{A^{\prime}}{A}\right)_{\Sigma} & =\left(\frac{\grave{v}}{\grave{v}}-\grave{v} \frac{m}{\mathrm{r}^{2}}+\grave{v} \frac{m_{\mathrm{r}}}{\mathrm{r}}\right)_{\Sigma}, \\
\left(\frac{Y Y^{\prime}}{B}\right)_{\Sigma} & =\left(\grave{v}\left(1-\frac{2 m}{\mathrm{r}}\right)-\mathrm{r} \grave{r}\right)_{\Sigma} .
\end{aligned}
$$

The gravitational mass function of the radiating star $m(v, r)$ can be obtained by eliminating $v, \grave{v}$ and $\grave{r}$ from Eq. (58b) above. It is given by

$$
m(v, r)=\left[\frac{Y}{2}\left(1+\frac{\dot{Y}^{2}}{A^{2}}-\frac{Y^{\prime 2}}{B^{2}}\right)\right]_{\Sigma},
$$

and is expressed only in terms of the metric potentials $A, B$ and $Y$. Following the modus operandi of the previous sections, we finally arrive at the following condition at the stellar surface 


$$
\begin{aligned}
& \frac{2 \dot{Y}^{\prime}}{A B Y}-2 \frac{\dot{B} Y^{\prime}}{A B^{2} Y}-2 \frac{A^{\prime} \dot{Y}}{A^{2} B Y}=2 \frac{m_{\mathrm{r}}}{\mathrm{r}^{2}} \\
& +\frac{1}{A^{2}}\left(-2 \frac{\ddot{Y}}{Y}-\frac{\dot{Y}^{2}}{Y^{2}}+2 \frac{\dot{A} \dot{Y}}{A Y}\right)+\frac{1}{B^{2}}\left(\frac{Y^{\prime 2}}{Y^{2}}+2 \frac{A^{\prime} Y^{\prime}}{A Y}\right)-\frac{1}{Y^{2}} .
\end{aligned}
$$

Observe that the result (60) is a shearing generalisation of the shear-free equation (31) to which it reduces to when $\sigma=0$. On simplifying (60), we arrive at

$$
\left(p+\epsilon-\mu+\frac{2}{3} \Delta\right)_{\Sigma}=\left(q+\epsilon-2 \frac{m_{\mathrm{r}}}{\mathrm{r}^{2}}\right)_{\Sigma},
$$

where we have utilised the field equations (55b) and (55d). The above then reduces to

$\left.p\right|_{\Sigma}=\left(q+\mu-\bar{\mu}-\frac{2}{3} \Delta\right)_{\Sigma}$.

Here we note the presence of the anisotropy term $\Delta=p_{\|}-$ $p_{\perp}$. The isotropic pressure is balanced by the interior heat flux $q$, and null string energy density $\mu$, the exterior null string density $\bar{\mu}$ and the anisotropy $\Delta$. The Eqs. (57), (59) and (61) are the most general conditions for matching the interior $\mathscr{M}^{-}$to the exterior $\mathscr{M}^{+}$across a comoving surface $\Sigma$.

We can state our result as follows:

Theorem Consider two four-dimensional spacetime manifolds $\mathscr{M}^{ \pm}$connected by the hypersurface $\Sigma$. The interior spacetime $\mathscr{M}^{-}$is described by the general spherically symmetric metric with a matter field containing a combination of a barotropic fluid, null dust and a null string fluid. The exterior spacetime $\mathscr{M}^{+}$is described by the generalised Vaidya metric containing null dust and a null string fluid. The boundary condition at the stellar surface $\Sigma$ is then

$\left.p\right|_{\Sigma}=\left(q+\mu-\bar{\mu}-\frac{2}{3} \Delta\right)_{\Sigma}$,

relating the isotropic pressure $p$ to the heat flux $q$, the anisotropy $\Delta$, the internal null string density $\mu$ and the external null string density $\bar{\mu}$.

It is important to emphasise that this theorem holds in the presence of both shear and anisotropy for a generalised composite matter distribution.

Analogous to the points presented in Sect. 4, several previous results are contained in the boundary condition (61). When $\Delta=0$, and the spacetime is shear-free, Eq. (61) reduces to the expression for (32) given by

$(p)_{\Sigma}=(q+\mu-\bar{\mu})_{\Sigma}$.

Other possible combinations of the matter variables $q, \mu, \bar{\mu}$ and $\Delta$, are presented in Table 1 .

\section{Discussion}

In this paper we derived the junction conditions for a radiating star with a composite matter distribution. We made the requirement that the interior and exterior fluid distributions be physically tractable and that the matching takes place across a comoving hypersurface. A shear-free interior spacetime with a composite matter distribution was matched smoothly to an exterior radiating geometry which is the generalised Vaidya atmosphere. It was found that our approach leads to the result

$p_{\Sigma}=(q+\mu-\bar{\mu})_{\Sigma}$

which is a generalisation of the Santos [1] boundary condition, as well as the condition derived by Maharaj et al. [32], with a clear physical interpretation. The pressure at the surface of the radiating star depends on the magnitude of the heat flux $q$ as well as the difference $\mu-\bar{\mu}$ of the null string densities from the two manifolds $\mathscr{M}^{-}$and $\mathscr{M}^{+}$, respectively. The junction condition (62) above is, as far as we aware, a new result and may become applicable to physically important astrophysical scenarios as the effects of the two null string energy densities $\mu$ and $\bar{\mu}$ are now included in the dynamics. These results were extended to arbitrary dimensions in the absence of shear, and boundary condition (62) holds for all dimensions greater than four. Finally, the general junction condition on the surface of a relativistic radiating star having an interior stellar fluid with nonzero shear and anisotropic stresses was presented. It was shown that the matching of the interior spacetime geometry to that of the generalised Vaidya exterior on the stellar boundary yielded

$\left.p\right|_{\Sigma}=\left(q+\mu-\bar{\mu}-\frac{2}{3} \Delta\right)_{\Sigma}$.

This is an important result which highlights the effects of the heat flux $q$, the two null string density components $\mu$ and $\bar{\mu}$ (pertaining to the internal matter of the star and the external atmosphere, respectively), as well as the degree of anisotropy $\Delta$, on the internal dynamics. The boundary condition (63) holds for a general spherical spacetime in general relativity.

In order to gauge and study the dynamical evolution of a relativistic radiating star with a composite matter distribution, the boundary condition needs to be solved for the matter variables. Both conditions (62) and (63) constitute new differential equations which are consistency equations, and need to be solved on the surface $\Sigma$ in order to yield radiating models. Particular solutions have been found in the past for barotropic matter distributions, see e.g. the model of de Oliveira and Santos [2]. We now have to include the generalised matter distribution (51) to generate a solution. This will be the basis for future work. 
Table 1 Isotropic pressure values on the surface $\Sigma$

\begin{tabular}{|c|c|c|c|c|}
\hline $\begin{array}{l}\text { Heat flux } \\
q\end{array}$ & $\begin{array}{l}\text { Internal null string density } \\
\mu\end{array}$ & $\begin{array}{l}\text { External null string density } \\
\bar{\mu}\end{array}$ & $\begin{array}{l}\text { Degree of anisotropy } \\
\Delta\end{array}$ & $\begin{array}{l}\text { Isotropic pressure } \\
\left.p\right|_{\Sigma}\end{array}$ \\
\hline Nonzero & Nonzero & Nonzero & 0 & $q+\mu-\bar{\mu}$ \\
\hline 0 & 0 & 0 & Nonzero & $-\frac{2}{3} \Delta$ \\
\hline Nonzero & 0 & 0 & Nonzero & $q-\frac{2}{3} \Delta$ \\
\hline 0 & Nonzero & 0 & Nonzero & $\mu-\frac{2}{3} \Delta$ \\
\hline 0 & 0 & Nonzero & Nonzero & $-\bar{\mu}-\frac{2}{3} \Delta$ \\
\hline Nonzero & 0 & Nonzero & Nonzero & $q-\bar{\mu}-\frac{2}{3} \Delta$ \\
\hline Nonzero & Nonzero & 0 & Nonzero & $q+\mu-\frac{2}{3} \Delta$ \\
\hline 0 & Nonzero & Nonzero & Nonzero & $\mu-\bar{\mu}-\frac{2}{3} \Delta$ \\
\hline
\end{tabular}

\section{Appendix A: Junction conditions}

Junction conditions have been extensively analysed to study astrophysical objects in general relativity. Consider two fourdimensional (bulk) spacetime manifolds $\mathscr{M}^{ \pm}$with oriented boundaries $\Sigma^{ \pm}$such that there exists a diffeomorphism between $\Sigma^{-}$and $\Sigma^{+}$. Each is endowed with symmetric, nondegenerate, smooth metric tensor fields $\mathbf{g}^{ \pm}$. Upon successful matching, the resulting spacetime $(\mathscr{M}, \mathbf{g})$ is the disjoint union of the spacetimes $\mathscr{M}^{ \pm}$with points on their oriented boundaries $\Sigma^{ \pm}$such that the junction conditions are satisfied [51]. Individual points on $\mathscr{M}^{ \pm}$are labelled by $\left\{x_{ \pm}^{a}\right\}$ where $a \in\{0, \ldots, 3\}$. Let $g_{i j}$ be the intrinsic metric to $\Sigma^{ \pm}$so that

$d s_{\Sigma^{ \pm}}^{2}=g_{i j} d \xi^{i} d \xi^{j}$.

The intrinsic coordinates to $\Sigma^{ \pm}$are given by $\left\{\xi_{ \pm}^{i}\right\}$ where $i \in\{1, \ldots, 3\}$. The two embeddings are given by the maps

$$
\begin{aligned}
& \Psi^{ \pm}: \Sigma \longrightarrow \mathscr{M}^{ \pm}, \\
& \xi^{i} \mapsto x_{ \pm}^{a}=\Psi_{ \pm}^{i}\left(\xi^{i}\right),
\end{aligned}
$$

such that $\Sigma^{ \pm} \equiv \Psi^{ \pm}(\Sigma) \subset \mathscr{M}^{ \pm}$. The diffeomorphism from $\Sigma^{+}$to $\Sigma^{-}$is $\Psi^{-} \circ \Psi^{+-1}$. The metrics in the regions $\mathscr{M}^{ \pm}$ are of the form

$d s_{ \pm}^{2}=g_{a b} d \chi_{ \pm}^{a} d \chi_{ \pm}^{b}$,

where $\chi_{ \pm}^{a}$ are the coordinates in $\mathscr{M}^{ \pm}$with $a \in\{0, \ldots, 3\}$. The requirement here is that the metrics (A.1) and (A.3) match smoothly across $\Sigma^{ \pm}$. This generates the first junction condition

$\left(d s_{-}^{2}\right)_{\Sigma^{-}}=\left(d s_{+}^{2}\right)_{\Sigma^{+}}=d s_{\Sigma}^{2}$,

which implies that $\Sigma^{-} \equiv \Sigma^{+}$, hence from here on both boundaries $\Sigma^{ \pm}$can be represented by $\Sigma$ which is embedded in a bulk spacetime. The above condition (A.4) is also referred to as the first fundamental form. The coordinates of $\Sigma$ on the spacetimes $\mathscr{M}^{ \pm}$are consequently given by $\chi_{ \pm}^{a}=\chi_{ \pm}^{a}\left(\xi_{ \pm}^{i}\right)$. The second junction condition is acquired by requiring the continuity of the extrinsic curvature of $\mathscr{M}^{ \pm}$across the boundary $\Sigma$. This results in

$K_{i j}^{-}=K_{i j}^{+}$,

where

$K_{i j}^{ \pm} \equiv-n_{a}^{ \pm} \frac{\partial^{2} \chi_{ \pm}^{a}}{\partial \xi_{ \pm}^{i} \partial \xi_{ \pm}^{j}}-n_{a}^{ \pm} \Gamma_{b c}^{a} \frac{\partial \chi_{ \pm}^{b}}{\partial \xi_{ \pm}^{i}} \frac{\partial \chi_{ \pm}^{c}}{\partial \xi_{ \pm}^{j}}$.

The above condition is called the second fundamental form where $n_{a}^{ \pm}\left(\chi_{ \pm}^{b}\right)$ are components of the vector normal to $\Sigma$. The junction conditions (A.4) and (A.5) are homologous with those generated by $[52,53]$.

Acknowledgements SDM and BPB thank the University of KwaZuluNatal for its continued support. SDM acknowledges that this work is based upon research supported by the South African Research Chair Initiative of the Department of Science and Technology and the National Research Foundation.

Data Availability Statement This manuscript has no associated data or the data will not be deposited. [Authors' comment: This is a theoretical study and the results can be verified from the information available.]

Open Access This article is licensed under a Creative Commons Attribution 4.0 International License, which permits use, sharing, adaptation, distribution and reproduction in any medium or format, as long as you give appropriate credit to the original author(s) and the source, provide a link to the Creative Commons licence, and indicate if changes were made. The images or other third party material in this article are included in the article's Creative Commons licence, unless indicated otherwise in a credit line to the material. If material is not included in the article's Creative Commons licence and your intended use is not permitted by statutory regulation or exceeds the permitted use, you will need to obtain permission directly from the copyright holder. To view a copy of this licence, visit http://creativecomm ons.org/licenses/by/4.0/.

Funded by $\mathrm{SCOAP}^{3}$.

\section{References}

1. N.O. Santos, Mon. Not. R. Astron. Soc. 216, 403 (1985)

2. A.K.G. de Oliviera, N.O. Santos, Astrophys. J. 312, 640 (1987) 
3. A. Banerjee, S.E.B. Choudhury, Gen. Relativ. Gravit. 21, 785 (1989)

4. R. Tikekar, L.K. Patel, Pramana J. Phys. 39, 17 (1992)

5. S.D. Maharaj, M. Govender, Pramana J. Phys. 54, 715 (2000)

6. M. Govender, S. Thirukkanesh, Int. J. Theor. Phys. 48, 3558 (2009)

7. S. Thirukkanesh, S. Moopanar, M. Govender, Pramana J. Phys. 79 , $223(2012)$

8. M.Z. Bhatti, Eur. Phys. J. Plus 131, 428 (2016)

9. Z. Yousaf, Eur. Phys. J. Plus 132, 71 (2017)

10. N.F. Naidu, M. Govender, K.S. Govinder, Int. J. Mod. Phys. D 15, 1053 (2006)

11. R. Chan, Mon. Not. R. Astron. Soc. 288, 589 (1997)

12. R. Chan, Mon. Not. R. Astron. Soc. 316, 588 (2000)

13. L. Herrera, N.O. Santos, Phys. Rev. D 70, 084004 (2004)

14. L. Herrera, A. Di Prisco, J. Ospino, Phys. Rev. D 76, 044001 (2006)

15. A. Di Prisco, L. Herrera, G. Le Denmat, M.A.H. MacCallum, N.O. Santos, Phys. Rev. D 76, 064017 (2007)

16. L. Herrera, A. Di Prisco, J. Ospino, Eur. Phys. J. C 80, 631 (2020)

17. L. Herrera, G. Le Denmat, N.O. Santos, A. Wang, Int. J. Mod. Phys. D 13, 583 (2004)

18. R. Sharma, S. Das, R. Tikekar, Gen. Relativ. Gravit. 47, 25 (2015)

19. B.C. Tewari, Gen. Relativ. Gravit. 45, 1547 (2013)

20. B.C. Tewari, K. Charan, Astrophys. Space Sci. 357, 107 (2015)

21. S.K. Maurya, Y.K. Gupta, Int. J. Theor. Phys. 52, 1075 (2013)

22. R.S. Bogadi, M. Govender, S. Moyo, Eur. Phys. J. C 135, 170 (2020)

23. J.M.Z. Pretal, Eur. Phys. J. C 80, 726 (2020)

24. N.F. Naidu, R.S. Bogadi, A. Kaisavelu, M. Govender, Gen. Relativ. Gravit. 52, 79 (2020)

25. M. Mars, J.M.M. Senovilla, Class. Quantum Gravity 10, 1865 (1993)

26. F. Fayos, J.M.M. Senovilla, R. Torres, Phys. Rev. D 54, 4862 (1996)

27. G.J. Olmo, D. Rubiera-Garcia, Class. Quantum Gravity 37, 215002 (2020)

28. Z. Yousaf, Eur. Phys. J. Plus 136, 281 (2021)

29. Z. Yousaf, Phys. Dark Universe 28, 100509(2020)
30. F.C. Mena, J.M. Oliveira, Ann. Phys. 387, 135 (2017)

31. K. Charan, O.P. Yadav, B.C. Tewari, Eur. Phys. J. C 81, 60 (2021)

32. S.D. Maharaj, G. Govender, M. Govender, Gen. Relativ. Gravit. 44, 1089 (2012)

33. V. Husain, Phys. Rev. D 53, 1759 (1996)

34. E.N. Glass, J.P. Krisch, Phys. Rev. D 57, 5945 (1998)

35. E.N. Glass, J.P. Krisch, Class. Quantum Gravity 16, 1175 (1999)

36. A.Z. Petrov, Gen. Relativ. Gravit. 32, 8 (2002)

37. A. Wang, Y. Wu, Gen. Relativ. Gravit. 31, 107 (1999)

38. B.P. Brassel, S.D. Maharaj, R. Goswami, Gen. Relativ. Gravit. 49, 37 (2017)

39. B.P. Brassel, R. Goswami, S.D. Maharaj, Phys. Rev. D 95, 124051 (2017)

40. J. Bekenstein, Phys. Rev. D 4, 2185 (1971)

41. B. Mashhoon, M. Partovi, Phys. Rev. D 20, 2455 (1979)

42. S.W. Hawking, G.F.R. Ellis, The Large Scale Structure of Spacetime (Cambridge University Press, Cambridge, 1973)

43. G. Govender, B.P. Brassel, S.D. Maharaj, Eur. Phys. J. C 75, 324 (2015)

44. E.N. Glass, J. Math. Phys. 31, 1974 (1990)

45. B. Bhui, S. Chaterjee, A. Banerjee, Astrophys. Space Sci. 226, 7 (1995)

46. A. Banerjee, S. Chaterjee, Astrophys. Space. Sci. 299, 219 (2005)

47. H. Shah, Z. Ahmed, S. Khan, Can. J. Phys. 96, 1163 (2018)

48. M.D. Mkenyeleye, R. Goswami, S.D. Maharaj, Phys. Rev. D 42, 024041 (2015)

49. S. Thirukkanesh, S.S. Rajah, S.D. Maharaj, J. Math. Phys. 53, 032506 (2012)

50. N.F. Naidu, M. Govender, S.D. Maharaj, Eur. Phys. J. C 78, 48 (2018)

51. W. Israel, Il Nuovo Cimento 44, 1 (1966)

52. S. O'Brien, J.L. Synge, Dublin Inst. Adv. Stud. A9, 1 (1952)

53. A. Lichnerowicz, Théories Relativistes de La Gravitation et de l'electromagnétisme (Masson, Paris, 1955) 